\section{Reply to C.J. Sweeney et al}

We thank Sweeney et al ${ }^{1}$ for their comments regarding our recent article in Journal of Clinical Oncology titled "Metastasis-Free Survival in Prostate Cancer: Faster Drug Approvals, Better Drugs?" 2 We agree that pricing of cancer therapeutics is a societal issue and that the benefits of a drug in preventing downstream consequences of prostate cancer (and subsequent use) must be considered.

Although our concerns with the metastasis-free survival (MFS) end point extend beyond pricing and monetary costs, it must be acknowledged that the rising costs of cancer drugs is a major health policy issue. ${ }^{3,4}$ Societal costs are greater when expensive drugs are administered to large populations of patients, some of whom could enjoy months to years of a high quality of life without taking that drug for a longer time. Although the authors cite "negotiated pricing" and other efforts to put pressure on drug costs, such strategies have not yet bent the cost curve for cancer therapeutics. $^{5}$

For this reason, costly drugs administered earlier in the disease course, as with apalutamide and enzalutamide in nonmetastatic castrate-resistant prostate cancer (nmCRPC), must demonstrate superiority to those same drugs administered later in the disease course, such as when a metastasis is detected on standard imaging. Clinically relevant end points such as health-related quality of life and overall survival are paramount. Sweeney et $\mathrm{al}^{1}$ cite data that real-world survival for metastatic CRPC has not significantly changed over the past decade as evidence that MFS is still relevant in the modern era. Although that is concerning, we must point out that these data are based on a small single-institution study, and the authors analyzed the impact of modern drugs only for patients diagnosed between 2010 and 2013. ${ }^{6}$ Abiraterone was approved for treatment of mCRPC in chemotherapy-naive men on the basis of a survival benefit compared with docetaxel in December 2012. Since 2013, numerous therapy options in the castrate-resistant and castrate-sensitive settings have been approved. National data in a current era may still reveal a longer median survival for metastatic CRPC. More importantly, whether novel antihormonal agents meaningfully improve this overall survival or other clinically relevant quality-of-life end points in nmCRPC is unclear and was not demonstrated in the SPARTAN (A Study of Apalutamide [ARN-509] in Men With Non-Metastatic Castration-Resistant Prostate Cancer) or PROSPER (Safety and Efficacy
Study of Enzalutamide in Patients With Nonmetastatic Castration-Resistant Prostate Cancer) trials., ${ }^{7,8}$ Although we hope that postmarketing studies will demonstrate this effect, apalutamide and enzalutamide were given full (regular) approval, and sponsors are not required to conduct such studies. Accelerated approvals are a commonly used mechanism to ensure that drugs demonstrating an improvement in a surrogate end point are available to patients but undergo postmarketing studies that confirm that such drugs meet uniform standards of clinical benefit. $^{9}$

We agree with and commend the authors on the rigor of the Intermediate Clinical Endpoints in Cancer of the Prostate (ICECaP) meta-analysis. ${ }^{10}$ In their correspondence, the authors mention several future analyses that may address our concerns with the MFS end point. We look forward to the mentioned follow-up analysis, potentially using more eligible trials with mature data conducted in a modern era of CRPC treatment, to validate the MFS end point. These future studies may also address our concerns about convenience data sampling in meta-analyses. Other confirmatory validation studies of MFS using real-world data sources may also provide this reassurance. As we mention in our article, MFS has biologic plausibility and at least some evidence of surrogacy, and the US Food and Drug Administration (FDA) Oncologic Drugs Advisory Committee concluded that MFS was an acceptable surrogate for nmCRPC. Furthermore, although we agree with the authors that nmCRPC and biochemically recurrent prostate cancer are biologically distinct entities and acknowledge this in our article, both entities were studied in the ICECaP meta-analysis validating the MFS end point, and MFS is being used as a primary end point in several adjuvant trials in high-risk prostate cancer. Data from these trials may be used to justify earlier initiation of costly systemic therapy.

Ultimately, oncologists want more treatment options that improve survival and/or quality of life for their patients with the least clinical and financial toxicity and therapeutic burden. Surrogate end points such as MFS ensure that more treatment options are available. However, it is paramount to demonstrate their intended benefit to avoid undue burden on patients and the health care system, particularly when such patients have years of high-quality life left to live. Drugs used earlier in the disease course should be held to a high evidence standard. Although generating this evidence is difficult, as the authors acknowledge, we do not believe that these 
goals are mutually exclusive or should disincentivize development of better therapies.

\section{Ravi B. Parikh, MD, MPP}

University of Pennsylvania, Philadelphia, PA

\section{Vinay Prasad, MD, MPH}

Oregon Health and Science University, Portland, OR

\section{AUTHORS' DISCLOSURES OF POTENTIAL CONFLICTS OF INTEREST AND DATA AVAILABILITY STATEMENT}

Disclosures provided by the authors and data availability statement (if applicable) are available with this article at DOI https://doi.org/10.1200/ JCO.19.00451.

\section{ACKNOWLEDGMENT}

Supported by National Institutes of Health training Grant No. 5-T32CA009615 (R.B.P.) and a grant from the Laura and John Arnold Foundation (V.P.)

\section{REFERENCES}

1. Sweeney CJ, De Abreu Lourenco R, Hamid AA, et al: What does metastasisfree survival actually mean? J Clin Oncol 37:1679-1680, 2019

2. Parikh RB, Prasad V: Metastasis-free survival in prostate cancer: Faster drug approvals, better drugs? J Clin Oncol 37:266-268, 2019
3. Meropol NJ, Schrag D, Smith TJ, et al: American Society of Clinical Oncology guidance statement: The cost of cancer care. J Clin Oncol 27: 3868-3874, 2009

4. Kantarjian H, Steensma D, Rius Sanjuan J, et al: High cancer drug prices in the United States: Reasons and proposed solutions. J Oncol Pract 10: e208-e211, 2014

5. Prasad V, De Jesús K, Mailankody S: The high price of anticancer drugs: Origins, implications, barriers, solutions. Nat Rev Clin Oncol 14:381-390, 2017

6. Francini E, Gray KP, Shaw GK, et al: Impact of new systemic therapies on overall survival of patients with metastatic castration-resistant prostate cancer in a hospital-based registry. Prostate Cancer Prostatic Dis 10.1038/ s41391-018-0121-2 [epub ahead of print on January 14, 2019]

7. Smith MR, Saad F, Chowdhury S, et al: Apalutamide treatment and metastasis-free survival in prostate cancer. N Engl J Med 378:1408-1418, 2018

8. Hussain M, Fizazi K, Saad F, et al: Enzalutamide in men with nonmetastatic, castration-resistant prostate cancer. N Engl J Med 378: 2465-2474, 2018

9. Beaver JA, Howie LJ, Pelosof L, et al: A 25-year experience of US Food and Drug Administration accelerated approval of malignant hematology and oncology drugs and biologics: A review. JAMA Oncol 4:849-856, 2018

10. Xie W, Regan MM, Buyse M, et al: Metastasis-free survival is a strong surrogate of overall survival in localized prostate cancer. J Clin Oncol 35: 3097-3104, 2017

DOI: https://doi.org/10.1200/JC0.19.00451; Published at jco.org on May 3, 2019.

\section{Access the Latest Scientific Presentations With the 2019 Gastrointestinal Cancers Symposium Videos and Slides}

\section{Gastrointestinal} Cancers Symposium

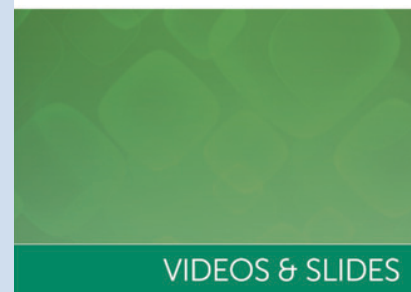

With the 2019 Gastrointestinal Cancers Symposium Videos and Slides, you can access practice-changing developments in gastrointestinal cancers care on any computer, tablet, or mobile device. Purchase the 2019 Gastrointestinal Cancers Symposium Slides separately or bundle the Videos and Slides and save at shop.asco.org

ASCO members save 20\%. Attendees of the Symposium receive the Videos and Slides for free. 
AUTHORS' DISCLOSURES OF POTENTIAL CONFLICTS OF INTEREST

\section{Reply to C.J. Sweeney et al}

The following represents disclosure information provided by authors of this manuscript. All relationships are considered compensated. Relationships are self-held unless noted. I = Immediate Family Member, Inst = My Institution. Relationships may not relate to the subject matter of this manuscript. For more information about ASCO's conflict of interest policy, please refer to www.asco.org/rwc or ascopubs.org/jco/site/ifc.

Ravi B. Parikh

Stock and Other Ownership Interests: Merck, Google, GNS Healthcare Consulting or Advisory Role: GNS Healthcare

Travel, Accommodations, Expenses: Conquer Cancer Foundation

\section{Vinay Prasad}

Patents, Royalties, Other Intellectual Property: Wrote a book titled "Ending Medical Reversal" and receive royalties from Johns Hopkins University Press. No other potential conflicts of interest were reported. 